Eight invited speakers and a large audience contributed to a lively discussion.

J. A. Brachet (Brussels), opening the morning session with a general review of the cytoplasm, emphasized that there is no such thing as a typical cell with a typical cytoplasm; rather, each cell type has a characteristically specialized cytoplasm. He also briefly reviewed the properties of cells in the absence of the nucleus.

The function of cytoplasmic components in plant cells was described by $\mathrm{D}$. H. Northcote (Cambridge). $\mathrm{He}$ showed that the Golgi bodies have different biochemical activities in different cell types and also that microtubules and endoplasmic reticulum act as guides in channelling the movement of materials within the cell. The problem of how intracellular events are coordinated was raised by Sir Rudolph Peters (Cam. bridge). Although he first posed this question 30 years ago, it still remains largely unanswered. Sir John Randall (London) presented the flagellum as an organelle that could be fruitfully used in studying morphopoiesis. One approach to this problem makes use of the finding that in the unicellular alga Chlamydomonas there are a number of mutants that map on the standard linkage groups which affect flagellar morphology and function. Another approach to the study of flagellar structure comes from following the course of flagellar regeneration. Two of Sir John's colleagues briefly presented biochemical and ultrastructural data on the flagella of mutant cells.

The afternoon session opened with a review of the structure and contents of mitochondria by M. Steinert (Brussels). He emphasized that in interpreting mitochondrial ultrastructure, one must be very wary of artefacts caused during their preparation for electron microscopy. Evidence for the presence of DNA, RNA and a protein synthesizing apparatus in mitochondria was also reviewed. The subject of the possible origin of mitochondria from bacteria arose, but it was pointed out in the discussion that followed the paper that we are too ready to look for similarities rather than for the differences between these two bodies. Peroxisomes were considered by C. de Duve (Louvain) to represent a primitive respiratory particle. He also described their interesting enzyme properties and their role in gluconeogenesis in plants and animals.

J. T. Dingle and A. J. Barrett (Cambridge) described experiments they had performed on inducing lysosomes of rat kidney cells to take up fluorescent dyes such as acridine orange. The uptake is passive and the lysosomes can concentrate and retain the dyes for many days. The way in which the dyes are bound is not known, nor is it understood what the significance of this finding is to the normal life of the cell. The afternoon was concluded by J. B. Gurdon (Oxford), who demonstrated how important the cytoplasm is in regulating the activity of the nucleus. Proof of this was provided from experiments with the frog, Xenopus, by transplanting nuclei from differentiated cells into egg cytoplasm. The cytoplasm imposes a decisive control over the nucleus regardless of the previous activity of the nucleus.

\section{Breeding Bacteria}

REPRESENTATIVES of eighteen countries met in Dubrovnik, Yugoslavia, for a two day meeting on "Genetics and Breeding of Streptomyces" from May 30 to June 1.
The symposium was sponsored by the Yugoslav Academy of Sciences and Arts and the Croatian Society for Microbiology, and was supported by Pliva Pharmaceutical and Chemical Works. The moeting came ten years after a first meeting on the same topic held in New York. As G. Sermonti remarked in his introductory words, plant and animal breeding have exploited cross-breeding procedures with great success, while bacterial (Streptomyces) breeding has got virtually no advantage from them, although simple methods were already available at the time of the first meeting.

The more important genetical procedures in Strepto. myces coelicolor A3(2) were reviewed by D. A. Hopwood, who also discussed the structure of zygotes and heteroclones in this species. Zygotes contain a complete chromosome from one parent and a random fragment from the other. By odd numbers of crossovers, zygotes give rise to terminally redundant structures replicating as such (heteroclones), while even numbers of cross. overs directly produce haploid recombinants. This last possibility was questioned by $G$. Sermonti, who assumed heteroclones to be a prerequisite to recombinant formation. The possible independent origin of zygotes and heterokaryons was also discussed, as well as the mating type situation in $S$. coelicolor. Fine structure genetic analysis in $S$. coelicolor has revealed the occurrence of a unit comprising five his genes and defined by two "hot spots" of crossover (A. Carere). An exhaustive review of the biology of actinophages was presented by $M$. Welsch, with special emphasis on lysogeny. Gene mapping in $S$. rimosus has been reported by M. Alačević. Cross-breeding in this species (S. Z. Mindlin) has, however, not yet profited by this information: markers introduced with parents do not affect yield of prototrophic recombinants; linkage between a streptomycin resistance locus and loci controlling tetracycline production was suggested.

Mutation-selection appears to be still the dominant procedure in Streptomyces breeding. A paper by R. J. Harold dealt with kinetic studies with 24 ultraviolet sensitive mutants, which map in two groups diametrically situated to one another in the chromosome of $S$. coelicolor. Of practical relevance was the report by $V$. Delić that up to 10 per cent auxotrophs can be obtained in Streptomyces by nitrosoguanidine at $p \mathrm{H} 9$.

Practical aspects of mutation-selection procedures were fully discussed. The antibiotic production spectrum of a mutagen-treated Streptomyces population is strictly dependent on the previous history of the strain. Highly productive strains are subjected more to negative mutations than to positive ones, the latter being obtained only with relatively low mutagen doses (S. I. Alikhanian). High producers of tetracycline can be found (E. L. Dulaney) among revertants from nonproducers, among auxotrophs and among back-mutants from auxotrophs.

On the whole, the symposium recorded well the ten years of progress in Streptomyces genetics, with their practical implications and applications. The dialogue between industrial microbiologists and geneticists, urged by $G$. Sermonti in his introductory words, did not, however, develop as hoped, partly because of the shortage of time, but certainly also because of excessive reticence on the industrial side. The next meeting should be in the USSR within the next five years. 